\title{
BMJ Open Quick-Wee versus bladder stimulation to collect midstream urine from precontinent infants under 1 year of age: a study protocol for a randomised controlled trial (ES.Stimquick.U)
}

To cite: Marchal S, Janicot J, Salicis J, et al. Quick-Wee versus bladder stimulation to collect midstream urine from precontinent infants under 1 year of age: a study protocol for a randomised controlled trial (ES.Stimquick.U). BMJ Open 2021;11:e046324. doi:10.1136/ bmjopen-2020-046324

- Prepublication history and additional supplemental material for this paper are available online. To view these files, please visit the journal online (http://dx.doi.org/10.1136/ bmjopen-2020-046324).

SM and JJ contributed equally.

Received 06 November 2020 Accepted 21 August 2021

D) Check for updates

(c) Author(s) (or their employer(s)) 2021. Re-use permitted under CC BY-NC. No commercial re-use. See rights and permissions. Published by BMJ.

For numbered affiliations see end of article.

Correspondence to

Dr Sarah Marchal;

marchal.s@pediatrie-chulenvalnice.fr

\section{ABSTRACT}

Introduction Urinary tract infections occur in around $1 \%-4 \%$ of boys and $3 \%-8 \%$ of girls under 2 years old. Diagnosis is difficult because of non-specific symptoms and the risk of urine analysis contamination depending on the sampling method used for precontinent infants. The American Academy of Pediatrics recommend transurethral catheterisation and suprapubic aspiration because of a low contamination rate but these techniques are invasive. On the other hand, while the National Institute for Health and Care Excellence advocate clean catch urine for its minimal invasiveness and acceptable contamination rate, it is difficult to accomplish in precontinent infants. Two recent methods have been described: the Quick-Wee method by Kaufman et al (suprapubic stimulation with cold salinesoaked gauze); and bladder stimulation by Herreros et al then by Tran et al (pubic tapping alternating with lumbar massage). This study aims to compare the effectiveness in collecting midstream urine by bladder stimulation vs the Quick-Wee method in infants under 1 year, before walking. Methods and analysis This study is a multicentre randomised controlled trial of 230 infants under 1 year and before walking who need urine analysis, conducted in four paediatric emergency departments in France. Patients will be randomised into two groups: bladder stimulation and Quick-Wee method.

The primary endpoint will be the success rate of voiding at least $2 \mathrm{~mL}$ of urine in less than $5 \mathrm{~min}$.

Secondary outcomes are the time to collect at least 2 $\mathrm{mL}$ of urine, comfort, quality of urine and the risk factors associated with failure of the two techniques.

Ethics and dissemination The study protocol was approved by the French national ethic committee (consultative committee of the protection of persons). The results of the study will be published in a peer-reviewed journal.

Trial registration number Clinical Trials Registry NCT04587999.

Date and protocol version identifier October 2020, V.1.
Strengths and limitations of this study

- Easy, quick and reliable technique for urine collection in precontinent infants.

- Ethical approach to propose two non-invasive urine collection methods.

- Randomised controlled trial is the best methodology to assess an intervention.

- Ideally, each infant should undergo the two techniques to limit the interindividual variability.

- It cannot be done in double blind

\section{INTRODUCTION}

Urinary tract infections (UTIs), mostly acute pyelonephritis, are common in infants with a prevalence in children under 2 years at around $1 \%-4 \%$ in boys and $3 \%-8 \%$ in girls. ${ }^{12}$

Although symptoms are not specific in infants (especially before 3 months), fever is the most common sign. ${ }^{3}$ A delay in diagnosis can lead to complications such as severe sepsis, renal scarring, renal failure and high blood pressure. ${ }^{4-7}$

Cytobacteriological urine examination (CBUE) is the only examination that provides a definite diagnosis of UTI. However, the bacteriuria threshold defining a UTI varies according to the sampling methods. ${ }^{89}$ It is known that urine is difficult to collect from precontinent infants, and can be easily contaminated, wrongly leading to a diagnosis of UTI leading to unnecessary treatment.

The method to collect urine depends on various clinical guidelines but also on the clinician's preference. 
The American Academy of Pediatrics ${ }^{8}$ (USA, 2011) recommends urethral catheterisation because of a very low contamination rate $(3.8 \%-14.3 \%),{ }^{10-14}$ but this technique is invasive. Similarly, suprapubic puncture has a low contamination rate of $0 \%-9.1 \%$ but is highly invasive. ${ }^{101114}$ On the other hand, a urine collection bag is non-invasive and easy to access, at the expense of being time consuming and resulting in a high contamination rate ranging from $26.6 \%$ to $62.8 \% .{ }^{10}{ }^{13-15}$ The National Institute for Health and Care Excellence ${ }^{9}$ (UK, 2007) recommends clean catch urine as it is minimally invasive and has a contamination rate of $5 \%-26 \%$ which is considered acceptable. ${ }^{10-12}{ }^{14-16}$ However, while this method is easy for potty-trained children, it involves waiting more than 1 hour in $58 \%$ of cases in precontinent infants. ${ }^{17}$

Before the age of 2 years, urination is triggered by the proprioceptive stimulus of bladder distension, and also by perineal skin stimulation or abdominal pressure (via the pudendal reflex). This reflex results in a modification of the detrusor and pelvic-perineal muscle tone, responsible for urination. ${ }^{1819}$

Based on this premise, two methods of collecting urine samples have recently been described in the literature: bladder stimulation consisting of pubic tapping alternating with lumbar massage first described by Herreros $e t a l^{2021}$ and then by Tran $e t a l^{22}$; and the Quick-Wee method by suprapubic cutaneous stimulation with cold saline-soaked gauze described by Kaufman et al. ${ }^{23}{ }^{24}$ In the Herreros et al study, the success rate in obtaining urine in newborns was $86 \%{ }^{20}$ In the Tran et al study, the success rate was $55.6 \%$ on the first attempt and $23.2 \%$ on the second with an average voiding time of $63.6 \mathrm{~s}$. The target population was infants under 2 years of age who were not yet walking. The success rate decreased significantly with weight and a high EVENDOL score, ${ }^{22}$ a validated tool which measures pain in children under 7 years. In the study by Kaufman et al, the success rate was $31 \%$ compared with the group without stimulation $(12 \%, \mathrm{p}<0.001)$ in infants under 1 year, regardless of age or sex. ${ }^{23}{ }^{24}$ However, the published success rates of the two methods are not comparable, because of different study selection criteria (Tran et al included infants under 2 years of age who were not yet walking while previous studies included children under 1 year), time of stimulation ( $3 \mathrm{~min}$ in the Tran $e t$ al study vs $5 \mathrm{~min}$ in previous studies) and number of attempts (two for Tran et al vs only one for others).

We hypothesise that bladder stimulation results in more successful collection of midstream urine than the Quick-Wee method in infants under 1 year of age, before walking. We have designed a randomised, multicentre, prospective clinical trial to assess the superiority of Bladder Stimulation over the Quick-Wee method to collect midstream urine.

\section{METHODS AND ANALYSIS \\ Study aim}

The aim of the trial is to compare the effectiveness of two non-invasive midstream urine collection methods in precontinent infants under 1 year of age, before walking: 'the Quick-Wee method' versus 'the Bladder Stimulation method'.

\section{Study design and setting}

The trial is a French national prospective, multicentre, open-label, randomised controlled trial. The population to be studied is infants under 1 year of age who are not yet walking.

This research will be conducted in four paediatric departments of the Inter-Hospitals Paediatricians of Infectious Diseases network in France: paediatric hospitals of Nice University Hospital-Lenval, paediatric nephrology and haemodialysis services of Nice University Hospital, Grasse Hospital Center, Antibes-Juan les Pins Hospital Center.

\section{Eligibility criteria}

Eligibility and exclusion criteria are listed in table 1.

\section{Intervention}

Both the methods require genital cleaning beforehand with Dakin using sterile compresses then rinsed with sterile water, which is part of the sterile clean catch urine collection cup kit.

The bladder stimulation technique consists of one caregiver holding the child under the armpits with the legs dangling in males and hips flexed in females. A nurse or technician then alternates between bladder stimulation manoeuvres: gentle tapping in the suprapubic area at a frequency of 100 taps per minute for $30 \mathrm{~s}$ followed

Table 1 List of eligibility and exclusion criteria

$\begin{array}{ll}\text { Eligibility criteria } & \text { Exclusion criteria } \\ \text { Infants under the age of } 1 \text { year. } & \text { Presence of vital distress signs. } \\ \text { Precontinent. } & \text { Before walking. } \\ \text { Suspected febrile urinary tract infection, uropathy, kidney disease or } \\ \text { metabolic disease, requiring a urine analysis. }\end{array}$



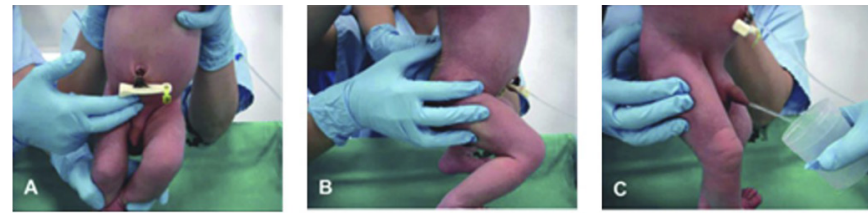

Figure 1 Description of the bladder stimulation method. (A) Gentle tapping in the suprapubic area at a frequency of 100 taps per minute; (B) lumbar paravertebral massage maneuvers; $(C)$ collecting midstream urine in a sterile container.

by lumbar paravertebral massage for $30 \mathrm{~s}$. The renal and bladder stimulation will last less than $5 \mathrm{~min}$. A third operator collects midstream urine in a sterile container (figure 1). ${ }^{22}$

The Quick-Wee method consists of stimulating the suprapubic area with circular movements with a cold and wet compress (soaked with $10 \mathrm{~mL}$ of $0.9 \% \mathrm{NaCl}$, kept in the refrigerator at $2.8^{\circ} \mathrm{C}$, and used within 2 min after removing from the refrigerator) held by sterile forceps. ${ }^{24}$

This results in the contraction of the detrusor via the parasympathetic nervous system and urination. Midstream urine is then collected in a sterile container. This method requires the presence of only one operator (figure 2).

In case of failure (less than $2 \mathrm{~mL}$ of urine after 5 min), urine will be collected by another technique

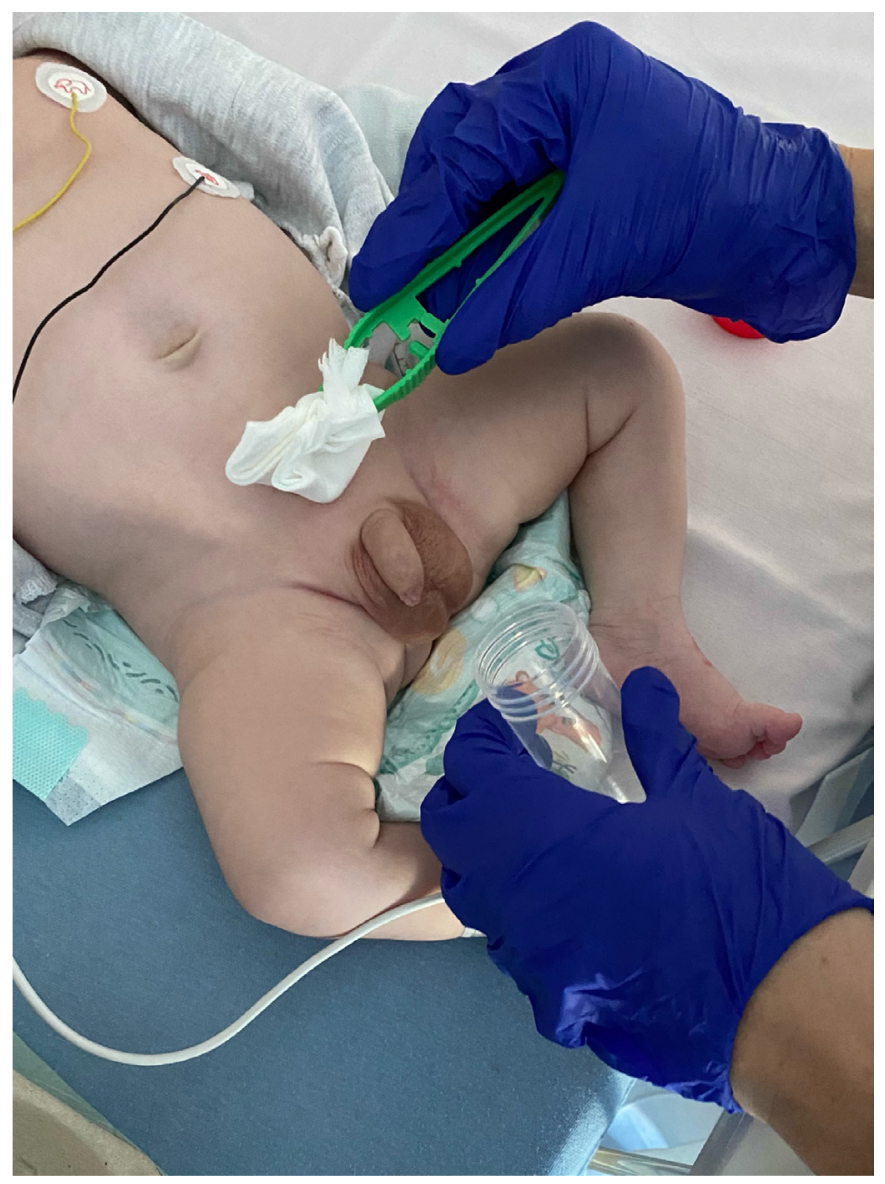

Figure 2 Description of the Quick-Wee method. (transurethral catheterisation, suprapubic aspiration or collection bag).

\section{Outcomes}

The primary outcome is the success rate of obtaining midstream urine defined as collecting at least $2 \mathrm{~mL}$ of urine in less than 5 min.

Secondary outcomes will assess the time required to obtain urine, the comfort during the procedure, the quality of the urine sample and identification of risk factors associated with failure of either of the techniques.

The time required to obtain urine is defined as the time between the start of the manoeuvres and urine collection after disinfection of the genitals. The techniques will only be performed once.

The time will be censored at $5 \mathrm{~min}$ in case of failure to collect urine or on abandonment of the procedure.

The comfort of each procedure is documented during the first $10 \mathrm{~s}$. Pain is evaluated using two scales: the EVENDOL (online supplemental appendix 1), ${ }^{25}$ and the FLACC (online supplemental appendix 2) ${ }^{26}$

The quality of the urine is determined by the degree of contamination which is defined by at least one of the following criteria: growth of two or more micro-organisms, presence of a non-uropathogenic germ (lactobacilli, coagulase negative staphylococcus, corynebacterium), leukocyturia under $10^{4} / \mathrm{mL}$, or bacteriuria between 0 and $10^{4}$ Colony Forming Unit $(\mathrm{CFU}) / \mathrm{mL}$. We will compare the bacterial contamination between the two techniques by calculating the ratio between the numbers of CBUE presenting a contamination over the number of CBUE carried out in each group. The number of failed attempts will also be indicated for each group.

Risk factors potentially associated with failure of the procedure (no urine or quantity under $2 \mathrm{~mL}$ after one attempt) that we will study are: age (in months), weight (in $\mathrm{kg}$ ), sex, pain at the beginning of procedure, presence of UTI, time since last meal (time between urine sample collection and whether last meal was more than 1 hour before or not), time since last urination (time between urine sample collection and whether last urination was more than 1 hour before or not).

\section{Participant timeline}

The study plan for enrolment, interventions and assessments for the infants are presented in table 2.

\section{Sample size}

Based on the literature data, ${ }^{24}$ the success rate for midstream urine collection by Quick-Wee is $31 \%$ in infants under 1 year of age. The success rate for midstream urine collection by bladder stimulation is $56 \%{ }^{22}$ Therefore, we plan to have a success rate of $50 \%$ in the Bladder Stimulation group.

Thus, the number of subjects per arm required to demonstrate this difference between the groups with a $\chi^{2}$ test would be 104 according to a bilateral hypothesis, at the alpha risk of $5 \%$ and with a power of $80 \%$ (nQuery 
Table 2 Schedule of enrolment, interventions and assessments: recommendations for interventional trials (SPIRIT)

\begin{tabular}{|c|c|c|c|c|c|c|}
\hline \multicolumn{2}{|l|}{ Study period } & \multirow{2}{*}{$\begin{array}{l}\text { Enrolment } \\
\mathrm{T}-\mathrm{X}\end{array}$} & \multirow{2}{*}{$\begin{array}{l}\text { Allocation } \\
\text { TO }\end{array}$} & \multicolumn{2}{|l|}{ Postallocation } & \multirow{2}{*}{$\begin{array}{l}\text { Close out } \\
T>48 \text { hours }\end{array}$} \\
\hline Timepoint & & & & $\begin{array}{l}\text { In the first } 10 \mathrm{~s} \text { of } \\
\text { the manoeuvre }\end{array}$ & $\begin{array}{l}\text { When urine is } \\
\text { collected }\end{array}$ & \\
\hline \multirow{2}{*}{ Enrolment } & Informed consent & $x$ & & & & \\
\hline & Randomisation & $x$ & & & & \\
\hline Interventions & Quick-Wee & & $x$ & & & \\
\hline \multirow[t]{3}{*}{ Assessments } & Efficacy to collect urine & & & & $\mathrm{X}$ & \\
\hline & Time to collect urine & & & & $x$ & \\
\hline & Comfort & & & $x$ & & \\
\hline
\end{tabular}

SPIRIT, Standard Protocol Items: Recommendations for Interventional Trials.

Advisor, V.7.0). We consider an additional $10 \%$ of patients to account for data loss. Therefore, a sample size of 230 infants under the age of 1 year will be included in the study (115 in each group).

According to our estimates, 250 patients per year who meet the inclusion criteria consult in the emergency departments of the investigating centres. Considering a participation rate of around $60 \%, 150$ infants are eligible per year. It seems reasonable to consider a study period of 18 months.

\section{Training Investigators to study}

The participating investigators will attend two training sessions on a low fidelity infant mannequin to ensure that the two methods are carried out identically. The first will be held 2 months before the start of the study after which the investigators will train the nursing staff. A second meeting to consolidate the training will then be held 1 month before the start of the study to answer any practical questions.

A video of Quick-Wee method is available (online supplemental appendix 3), concerning bladder stimulation, a video has already been published by Tran $e t ~ a l .{ }^{22}$ We will show these videos during the training.

\section{Recruitment}

Emergency department nursing and medical staff will recruit patients according to the eligibility criteria. Each parent of a child meeting the eligibility criteria will be given full oral information by an investigator and a written briefing note. After a period of reflection, the informed consent will be signed by one of the two parents or the holder of parental authority (online supplemental appendix 4). Recruitment will start in November 2020, and it is anticipated that it will be completed within 18 months. The duration of patient participation will be
1 day (time spent in the emergency department). The total duration of the study will be 24 months (including 6 months of data processing and statistical analysis).

\section{Allocation}

The patients will be randomly assigned into one of the two study groups in a 1:1 ratio: Bladder stimulation and Quick-Wee (figure 3). Randomisation will be performed centrally at the Department of Clinical Research and Innovation of Nice University Hospital (DRCI) (using the nQuery Advisor V.7.0 software). The patient's inclusion number and the arm will be communicated to the investigators automatically (inclusion randomisation module) and quickly (around the clock and 7 days a week). Owing to the nature of the intervention, nobody will be blinded after assignment to the intervention.

\section{Data collection methods}

Study data will be collected by means of a case report form (CRF) designed by the clinical research associate (CRA) in charge of the promotion of the study and the data manager of the DRCI of the University Hospital of Nice, under the responsibility of the principal investigator.

Demographic and patient data will include age and sex of patient, relevant medical comorbidities, previous UTI, known anatomical or neurological abnormality affecting voiding, reason for presentation based on triage code and clinical indication for urine collection and reasons for exclusions and refusals.

Clinical data will include all the outcomes described in the corresponding chapter.

\section{Data management}

Study data will be recorded in an electronic CRF (e-CRF) from the finalised paper CRF by the data manager of the DRCI with the OpenClinica software. The e-CRF will be 


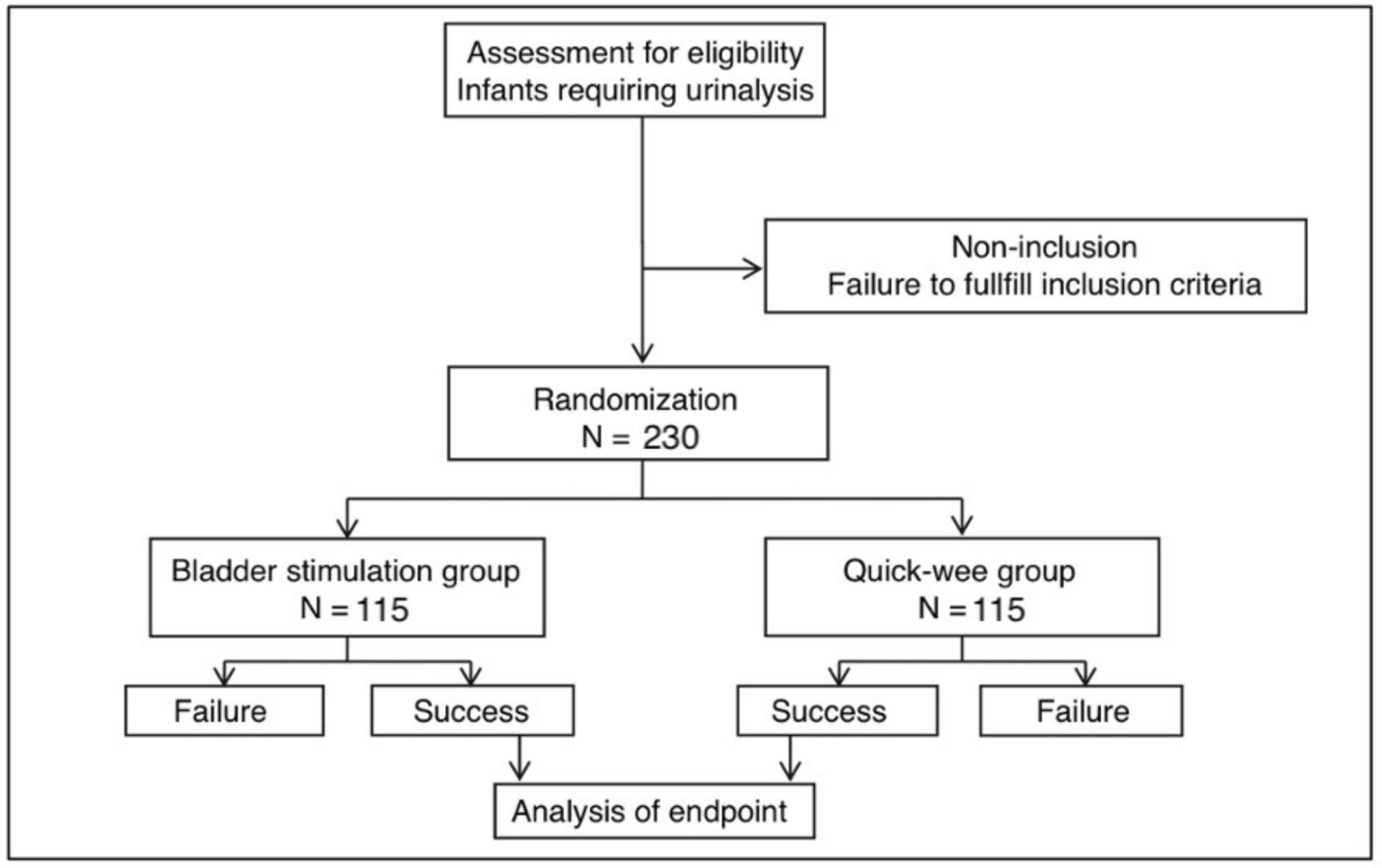

Figure 3 Flow chart.

set up and implemented under the responsibility of the DRCI. The investigators will capture the data collected during the study directly into the e-CRF with the assistance of the study CRA. Data security will be ensured by the creation of specific access rights according to the role of each professional participating in the study. Data quality control will be organised on the e-CRF during the monitoring visits planned by CRA promoters of the DRCI. At the end of the process, the database will be frozen and transferred for statistical analysis.

\section{Statistical methods}

Statistical analyses will be conducted using SAS Enterprise Guide V.7.1 software (Copyright (c) 2017 by SAS Institute). As advised in the Consolidated Standards of Reporting Trials guidelines, the main patient characteristics will be compared between the two groups from a clinical and non-statistical point of view. In one or more of the patient characteristics are not well matched between the groups, analysis can be adjusted for this variable.

Patients in the study will undergo an intention-to-treat analysis: each patient will be analysed in the assigned group by randomisation. Due to the brevity of patient participation in the study, it is not expected that patients will be lost to follow-up although there is a possibility of missing data due to technical problems.

The primary outcome is the success rate of obtaining a urine sample and will be calculated as a percentage by calculating the ratio between the number of successful procedures and the total number of procedures. The groups will be compared by a Cochran-Mantel-Haenszel $\chi^{2}$ test with the centre as the adjustment variable.

The secondary outcomes are time to urine collection, comfort, contamination and risk factors in case of failure.
Time to urine collection will be plotted as Kaplan-Meier curves and the two groups will be compared using the logrank test. A multivariate analysis will be performed using a Cox model to stratify parameters of randomisation (centre). The EVENDOL (EValuation ENfant DOuLeur) and FLACC (Face Legs Activity Cry Consolability) pain scores in the two groups will be compared using a MannWhitney non-parametric comparison of means test. The contamination rate will be compared between the groups by a $\chi^{2}$ test and adapted if necessary to small samples (Fisher's exact test). Finally, the risk factors for failed urine collection will be carried out using logistic regression models. ORs and their 95\% CIs will be presented.

\section{Data monitoring and auditing}

No interim analysis will be undertaken, and recruitment will continue until enrolment is completed. A data monitoring committee is not required for this low-risk study.

\section{Harms}

Minor temporary discomfort to the child could be caused by cold saline cutaneous stimulation or by holding the infants under their armpits (crying, mild distress). Even if it is considered exceptional, there is a risk of accidental fall of the infants. All complications in relation to the bladder stimulation or the Quick-Wee method will be documented during the trial and reported to the sponsor without delay. These data will be provided for the periodical review of a data and safety monitoring board.

\section{Outlook and significance}

Patients participating in the study will benefit from a non-invasive technique, performed in a reasonable time and with a low rate of contamination. If our hypothesis 
holds true, the bladder stimulation technique could be extended to other emergency departments and laboratories in the future. Moreover, it would reduce the additional costs generated by equipment, paraclinical exams and unnecessary treatments.

\section{Limitations}

A potential bias from the investigator could arise as the trial is non-blinded. However, given the procedures involved, it is not possible to run a blinded study. Ideally, each infant should undergo the two techniques to limit the interindividual variability but this would be difficult, time-consuming and unethical. Finally, we could compare the bladder stimulation technique with transurethral catheterisation which is currently the gold standard. However, we prefer to compare two non-invasive ways of sampling urine.

\section{Current status}

Study enrolment will start in November 2020. Recruitment and data analysis are expected to be completed by May 2022.

\section{ETHICS AND DISSEMINATION}

\section{Protocol amendments}

Protocol amendments will be updated and available on Clinical Trial registry website.

\section{Consent or assent}

Parents will be given a parent/guardian information sheet (PGIS). Participation in the study will be discussed with the clinician and/or researcher. This will take place in the emergency department. The parents will be assured that their care will not be affected if they do not wish their infants to participate. This is also stated in the PGIS. Verbal consent obtained from the parent/guardian (prior to undertaking sample collection) will be documented by nursing and medical staff. Refusal of consent will be recorded.

\section{Confidentiality}

Personal information about potential and enrolled participants will be stored in a password-protected database protected by a password known by the research team only.

\section{Dissemination policy}

Results will be written up in a thesis and published in a peer-reviewed journal.

\section{Trial status}

This trial protocol is published using V.1 on October 2020 as approved by a National Ethics Committee. Recruitment will start on November 2020 and is estimated to be completed in May 2022.

\footnotetext{
Author affiliations

${ }^{1}$ Service des Urgences Pédiatriques, Hôpitaux Pédiatriques de Nice CHU-LENVAL, Nice, France

${ }^{2}$ Service de Pédiatrie, Centre Hospitalier de Grasse, Grasse, France
}

${ }^{3}$ Service de Pédiatrie, Centre Hospitalier Princesse Grace, Monaco

${ }^{4}$ Service de Néphrologie Pédiatrique, Centre Hospitalier Universitaire de Nice, Nice, France

${ }^{5}$ Service de Chirurgie Pédiatrique, Hôpitaux Pédiatriques de Nice CHU-LENVAL, Nice, France

${ }^{6}$ Délégation à la Recherche Clinique et à l'Innovation, Centre Hospitalier Universitaire de Nice, Nice, France

${ }^{7}$ Service de Pédiatrie, Centre Hospitalier d Antibes Juan les Pins, Antibes, France

Acknowledgements The authors thank the families and clinical staff participating in this trial.

Contributors AT, SM, JS and JJ identified the research question, elaborated the study design and development of the protocol, drafted this paper and approved the final manuscript. DDo contributed to the development of the protocol. DDe, A-LH, $\mathrm{MO}, \mathrm{AR}, \mathrm{HH}, \mathrm{EB}, \mathrm{JBr}$, JBe, CR, EF, JD and CS-F contributed as coinvestigators for the study. EF participated in the development of the protocol and will be responsible for the statistical analysis.

Funding This work was supported by the Department of Clinical Research and Innovation of Nice University Hospital (grant number: 2020-A02303-36). A funding of $€ 13000$ has been granted. Contact information of the trial sponsor: 57 avenue de la Californie, 06200, Nice. Phone number: 04.92.03.03.92.

Competing interests None declared.

Patient consent for publication Parental/guardian consent obtained.

Provenance and peer review Not commissioned; externally peer reviewed.

Supplemental material This content has been supplied by the author(s). It has not been vetted by BMJ Publishing Group Limited (BMJ) and may not have been peer-reviewed. Any opinions or recommendations discussed are solely those of the author(s) and are not endorsed by BMJ. BMJ disclaims all liability and responsibility arising from any reliance placed on the content. Where the content includes any translated material, BMJ does not warrant the accuracy and reliability of the translations (including but not limited to local regulations, clinical guidelines, terminology, drug names and drug dosages), and is not responsible for any error and/or omissions arising from translation and adaptation or otherwise.

Open access This is an open access article distributed in accordance with the Creative Commons Attribution Non Commercial (CC BY-NC 4.0) license, which permits others to distribute, remix, adapt, build upon this work non-commercially, and license their derivative works on different terms, provided the original work is properly cited, appropriate credit is given, any changes made indicated, and the use is non-commercial. See: http://creativecommons.org/licenses/by-nc/4.0/.

\section{ORCID iDs}

Jade Janicot http://orcid.org/0000-0002-9976-7951

Antoine Tran http://orcid.org/0000-0002-1836-2742

\section{REFERENCES}

1 Bachur R, Harper MB. Reliability of the urinalysis for predicting urinary tract infections in young febrile children. Arch Pediatr Adolesc Med 2001;155:60-5.

2 Hoberman A, Chao HP, Keller DM, et al. Prevalence of urinary tract infection in febrile infants. J Pediatr 1993;123:17-23.

3 National Collaborating Centre for Women's and Children's Health (UK). Urinary tract infection in children: diagnosis, treatment and long-term management. London: RCOG Press, 2007.

4 Pitetti RD, Choi S. Utility of blood cultures in febrile children with UTI. Am J Emerg Med 2002;20:271-4.

5 Schnadower D, Kuppermann N, Macias CG, et al. Febrile infants with urinary tract infections at very low risk for adverse events and bacteremia. Pediatrics 2010;126:1074-83.

6 Shaikh N, Mattoo TK, Keren R, et al. Early antibiotic treatment for pediatric febrile urinary tract infection and renal scarring. JAMA Pediatr 2016;170:848.

7 Toffolo A, Ammenti A, Montini G. Long-Term clinical consequences of urinary tract infections during childhood: a review. Acta Paediatr 2012;101:1018-31

8 Subcommittee on Urinary Tract Infection, Steering Committee on Quality Improvement and Management, Roberts KB. Urinary tract infection: clinical practice guideline for the diagnosis and management of the initial UTI in febrile infants and children 2 to 24 months. Pediatrics 2011;128:595-610. 
9 Baumer JH, Jones RWA. Urinary tract infection in children, National Institute for health and clinical excellence. Archives of Disease in Childhood - Education and Practice 2007;92:189-92.

10 Tosif S, Baker A, Oakley E, et al. Contamination rates of different urine collection methods for the diagnosis of urinary tract infections in young children: an observational cohort study. J Paediatr Child Health 2012;48:659-64.

11 Teo S, Cheek JA, Craig S. Improving clean-catch contamination rates: a prospective interventional cohort study: improving cleancatch contamination rates. Emerg Med Australas 2016;28:698-703.

12 Herreros ML, Tagarro A, García-Pose A, et al. Performing a urine dipstick test with a clean-catch urine sample is an accurate screening method for urinary tract infections in young infants. Acta Paediatr 2018;107:145-50.

13 Al-Orifi F, McGillivray D, Tange S, et al. Urine culture from bag specimens in young children: are the risks too high? J Pediatr 2000;137:221-6.

14 Karacan C, Erkek N, Senel S, et al. Evaluation of urine collection methods for the diagnosis of urinary tract infection in children. Med Princ Pract 2010;19:188-91.

15 Alam MT, Coulter JBS, Pacheco J, et al. Comparison of urine contamination rates using three different methods of collection: clean-catch, cotton wool pad and urine bag. Ann Trop Paediatr 2005;25:29-34.

16 Altuntas N, Tayfur AC, Kocak M, et al. Midstream clean-catch urine collection in newborns: a randomized controlled study. Eur $\mathrm{J}$ Pediatr 2015; 174:577-82.

17 Davies P, Greenwood R, Benger J. Randomised trial of a vibrating bladder stimulator--the time to pee study. Arch Dis Child 2008;93:423-4.
18 Bréaud J, Oborocianu I, Bastiani F, et al. [Voiding disorders in childhood: from physiology to symptomatology]. Arch Pediatr 2012;19:1226-30.

19 Woock JP, Yoo PB, Grill WM. Mechanisms of reflex bladder activation by pudendal afferents. Am J Physiol Regul Integr Comp Physiol 2011;300:R398-407.

20 Herreros Fernández ML, González Merino N, Tagarro García A, et al. A new technique for fast and safe collection of urine in newborns. Arch Dis Child 2013;98:27-9.

21 Herreros ML, Tagarro A, García-Pose A, et al. Accuracy of a new clean-catch technique for diagnosis of urinary tract infection in infants younger than 90 days of age. Paediatr Child Health 2015;20:e30-2.

22 Tran A, Fortier C, Giovannini-Chami L, et al. Evaluation of the bladder stimulation technique to collect midstream urine in infants in a pediatric emergency department. PLoS One 2016;11:e0152598.

23 Kaufman J, Fitzpatrick P, Tosif S, et al. The QuickWee trial: protocol for a randomised controlled trial of gentle suprapubic cutaneous stimulation to hasten non-invasive urine collection from infants. BMJ Open 2016;6:e011357.

24 Kaufman J, Fitzpatrick P, Tosif S, et al. Faster clean catch urine collection (Quick-Wee method) from infants: randomised controlled trial. BMJ 2017;357:j1341.

25 Fournier-Charrière $\mathrm{E}$, Tourniaire $\mathrm{B}$, Carbajal R, et al. EVENDOL, a new behavioral pain scale for children ages 0 to 7 years in the emergency department: design and validation. Pain 2012;153:1573-82.

26 Crellin DJ, Harrison D, Santamaria N, et al. The psychometric properties of the FLACC scale used to assess procedural pain. $J$ Pain 2018;19:862-72. 